OPEN ACCESS

Edited by:

Vincenzo Romei,

Università degli Studi di Bologna, Italy

Reviewed by:

Berthold Langguth,

University of Regensburg, Germany Peter Giacobbe,

Sunnybrook Health Sciences Centre,

Canada

*Correspondence:

Chris Baeken

chris.baeken@UGent.be

Specialty section:

This article was submitted to

Consciousness Research,

a section of the journal

Frontiers in Psychology

Received: 31 July 2018 Accepted: 01 October 2018

Published: 31 October 2018

Citation:

Baeken C (2018) Accelerated rTMS: A Potential Treatment to Alleviate Refractory Depression.

Front. Psychol. 9:2017. doi: 10.3389/fpsyg.2018.02017

\section{Accelerated rTMS: A Potential Treatment to Alleviate Refractory Depression}

\author{
Chris Baeken ${ }^{1,2,3 *}$ \\ ${ }^{1}$ Department of Psychiatry and Medical Psychology, Ghent University, Ghent, Belgium, ${ }^{2}$ Ghent Experimental Psychiatry \\ (GHEP) Lab, Ghent University, Ghent, Belgium, ${ }^{3}$ Department of Psychiatry, Vrije Universiteit Brussel - Universitair Ziekenhuis, \\ Brussels, Belgium
}

Three decades of clinical research on repetitive transcranial magnetic stimulation (rTMS) has yielded one clear treatment indication in psychiatry for major depression disorder (MDD). Although the clinical response equals the standard treatment algorithms, the effect sizes on the beneficial outcome remain rather modest. Over the last couple of years, to improve the efficacy in resistant depression, two new avenues have been developed: personalization and intensifying rTMS treatment. For the latter, we retrospectively compared accelerated high-frequency rTMS (arTMS) with accelerated intermittent theta burst stimulation (aiTBS) in the refractory depressed state. Although the clinical efficacy was not significantly different between both protocols, our observations substantiate the potential of the accelerated stimulation to shorten the treatment duration from the depressed state to the response state. Any time gain from the depressed state to the recovered state is in the patients' interest.

\section{Keywords: accelerated rTMS, HF-rTMS, theta-burst stimulation, major depressive disorder, refractory MDD}

\section{INTRODUCTION}

Three decades of clinical repetitive transcranial magnetic stimulation (rTMS) research has resulted in only one clear indication for the treatment of (moderate) medication-resistant major depression in the field of psychiatry, specifically when stimulating the left dorsolateral prefrontal cortex (DLPFC) (Lefaucheur et al., 2014). Despite the many parameter adjustments tested over the past years, the rTMS outcome rates remain rather modest (Brunoni et al., 2017), meaning that only 3 or 4 out of 10 patients undergoing rTMS are going to respond, but will not be necessarily remitted. Importantly, like electroconvulsive therapy (ECT), another widely used neurostimulation application for treating patients with major depressive disorder (MDD), the rTMS must also be considered as an acute treatment option with relatively fast but no definitive long-term clinical improvement. Electroconvulsive therapy is considered to be one of our most potent treatment modalities in the acute phase of depression, yielding "unchallenged" response rates when compared with the classic pharmacological and current rTMS treatment algorithms (Baeken et al., 2013). After approximately 10 sessions of unilateral or bilateral (bifrontal/bitemporal) ECT, 80-90\% of the treated depression patients will clinically respond (Kennedy and Giacobbe, 2007). Of note, when including the treatment-resistant depression (TRD) patients, the response rates tend to be a bit lower, which is around 50-70\% (Rush and Siefert, 2009). 
Over the last couple of years, to optimize the rTMS treatment efficacy, the individualization of rTMS parameters (Padberg et al., 2017) and intense rTMS paradigms are under investigation (Holtzheimer et al., 2010). For the first approach, to accurately target the stimulation area, individual anatomical data are included (Fitzgerald et al., 2009), individual brain connectivity patterns are selected (Fox et al., 2012), or depression "biotypes" based on the brain network analysis are targeted (Drysdale et al., 2017). The second approach, accelerated rTMS (arTMS) treatment, applies multiple daily sessions, reducing the total time period of stimulation by just over a couple of days. This is in contrast to the classical daily single rTMS sessions, which usually spread over 2-4 weeks and sometimes even longer (Holtzheimer et al., 2010; Baeken et al., 2015). It is of interest to note that when applied to the left DLPFC, no significant differences in remission or response rates or reductions in depression severity scores were observed between the accelerated and the classical daily rTMS treatments (Fitzgerald et al., 2018).

Newer stimulation parameters, which deliver pulses even on a shorter amount of time, compare the excitatory stimulation protocols of high-frequency rTMS (HF-rTMS) and intermittent theta-burst stimulation (iTBS), when applied to the dorsomedial prefrontal cortex (dmPFC). Bakker et al. (2015) did not find any differences in clinical outcome between these two protocols. Recently, in a large, randomized, non-inferiority trial with TRD patients, Blumberger et al. (2018) also reported no differences in the clinical outcome between HF-rTMS and iTBS treatments, when applied to the left DLPFC.

From a clinical perspective, it would be highly interesting if we could apply "intensified" rTMS treatment algorithms, resulting not only in fast clinical improvement but also in reaching the response and remission rates as observed with ECT. As mostly refractory depression patients are selected for ECT (besides psychotic and catatonic depression), we retrospectively compared our aHF-rTMS with accelerated iTBS (aiTBS) treatments in this research (Baeken et al., 2013; Duprat et al., 2016), by only selecting stage III TRD patients. We expected that in this refractory MDD sample, response and remission rates would not be significantly different between the patients receiving aHF-rTMS or aiTBS treatments. We also evaluated on the assumption that arTMS treatment would reach comparable remission rates [remission defined as a final score on the 17-item Hamilton Depression Rating Scale score $\leq 7$ (HDRS: Hamilton, 1967)], as observed with ECT.

\section{MATERIALS AND METHODS}

From the original samples of our former aHF-rTMS and aiTBS accelerated paradigms comprising of patients with varying stages of treatment resistance (Baeken et al., 2013; Duprat et al., 2016), we have included only rTMS naïve unipolar stage III TRD patients in this research (Rush et al., 2003), with a mean duration of their current depression episode of 6.26 years $(s d=8.29)$, confirming only to the refractory MDD (Ward and Irazoqui, 2010). As ECT non-response is a poor predictor of clinical response to rTMS, stage V TRD patients were not included (George and Post, 2011).

A total of 28 stage III TRD patients remained, with 14 in each group. According to the criteria of Rush et al. (2003), all patients had a minimum of two unsuccessful psychopharmaceutical treatment trials with a selective serotonin reuptake inhibitor (SSRI) or serotonin-norepinephrine reuptake inhibitor (SNRI), and one failed clinical trial with a tricyclic antidepressant (TCA). After a washout period, all patients were noted as antidepressantfree, at least 2 weeks before entering the studies. Exclusion criteria were current or history of epilepsy, neurosurgical interventions, metal objects in the brain, having a pacemaker, having had ECT, alcohol dependence, and suicide attempts within 6 months before the start of the study. Bipolar and psychotic depressed patients were also excluded. Following standard practice, clinical response was defined as a $50 \%$ reduction of the baseline 17 -item HDRS score (Hamilton, 1967) by certified psychiatrists not related to the studies.

In both accelerated sham-controlled crossover protocols, all refractory MDD patients were randomized to receive either active or sham stimulation (20 sessions in total, 5 sessions per day for 4 days) at $110 \%$ of the resting motor threshold. In the following week, the order was reversed. For aHF-rTMS $(20 \mathrm{~Hz})$ treatment, patients received 40 trains of $1.9 \mathrm{~s}$ duration, separated by an intertrain interval of $12 \mathrm{~s}$ (1560 pulses per session) (Baeken et al., 2013). For the accelerated iTBS treatment trial, patients received 40 trains ( 1620 pulses per session) in 54 triplet bursts with a train duration of $2 \mathrm{~s}$ and a cycling of $8 \mathrm{~s}$ period (Duprat et al., 2016). In both studies, we targeted exactly the same region of the left DLPFC under MRI guidance.

\section{RESULTS}

No significant differences on depression severity scores were observed between the two arTMS protocols. The 2 (Time; before vs. after treatment $) \times 2($ Group; aiTBS vs. HF-rTMS $) \times 2$ (Order; first active then sham vs. first sham then active) analysis of variance (ANOVA) with individual HDRS scores as dependent variables showed a significant main effect of Time $F(2,48)=15.98$, $p<0.01$ but not of Group $F(1,24)=0.02, p=0.88$ or Order $F(1,24)=0.22, p=0.64$. The two-way interactions between Group and Order, Time and Order, and Time and Group were all not significant $(p$ 's $>0.05)$. Also, the three-way interaction between Time, Order, and Group was not significant $F(2,48)=0.20$, $p=0.82$ (Figure 1). Separately mixed ANOVAs, separated for 1-2 weeks also showed non-significant results. Pearson $X^{2}$ showed no group differences in the remission rates $\left[X^{2}(1)=1.12, p=0.28\right]$. Also, no significant demographic differences were observed. See Table 1.

\section{DISCUSSION}

Aligned with the classical daily HF-rTMS/iTBS clinical outcome findings (Bakker et al., 2015; Blumberger et al., 2018), our observations with the accelerated aHF-rTMS/aiTBS treatment protocols suggest similar clinical effects. 
35

30

25

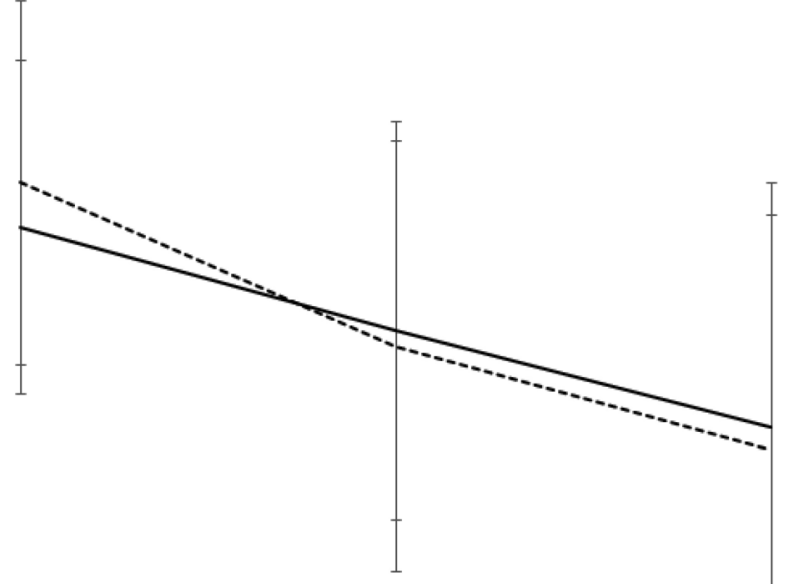

10

----aHF-rTMS — aiTBS

5

time

T1

$\mathrm{T} 2$

T3

FIGURE 1 | Graphical representation of the HDRS scores (mean and standard deviations) at baseline $\left(T_{1}\right)$, after 1-week $\left(T_{2}\right)$, and after 2-weeks of stimulation $\left(T_{3}\right)$ for aHF-rTMS and aiTBS treatment separately.

TABLE 1 | Demographic and outcome variables.

\begin{tabular}{|c|c|c|c|c|}
\hline & All & aHF-rTMS & aiTBS & $p$-values \\
\hline Number of patients & 28 & 14 & 14 & - \\
\hline Gender (F/M) & $14: 14$ & $8: 6$ & $6: 8$ & 0.45 \\
\hline Age & $48.37(11.74)$ & $47.86(13.58)$ & $48.86(10.05)$ & 0.83 \\
\hline Response $T_{3}$ (yes/no) & 10 & 7 & 3 & 0.12 \\
\hline Remission $\mathrm{T}_{3}$ (yes/no) & 4 & 3 & 1 & 0.28 \\
\hline Duration current depressive episode (years) & $6.26(8.29)$ & $7.89(8.03)$ & $4.62(8.51)$ & 0.31 \\
\hline Order $(R>S)$ & $9: 19$ & $6: 8$ & $3: 11$ & 0.23 \\
\hline Benzodiazepine intake (number) & 14 & 9 & 6 & 0.26 \\
\hline Benzodiazepine intake (mg/day) & $10.11(11.74)$ & $11.71(13.25)$ & $8.50(10.27)$ & 0.48 \\
\hline rMT (\%) & $59.86(6.27)$ & $60.00(4.98)$ & $59.71(7.54)$ & 0.91 \\
\hline $\mathrm{HDRS}_{1}$ & $24.07(6.10)$ & $24.86(6.42)$ & $23.29(5.89)$ & 0.51 \\
\hline $\mathrm{HDRS}_{2}$ & $19.36(7.21)$ & 19.07 (7.95) & $19.64(6.69)$ & 0.84 \\
\hline $\mathrm{HDRS}_{3}$ & $15.82(8.34)$ & $15.43(9.39)$ & $16.21(7.48)$ & 0.81 \\
\hline $\mathrm{HDRS}_{4}$ & - & - & $15.71(8.23)$ & - \\
\hline Response $T_{4}$ (yes/no) & - & - & 5 & - \\
\hline Remission $\mathrm{T}_{4}$ (yes/no) & - & - & 3 & - \\
\hline
\end{tabular}

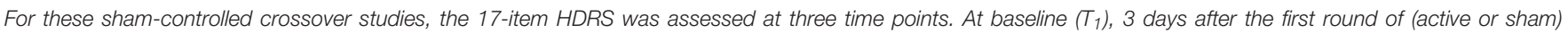

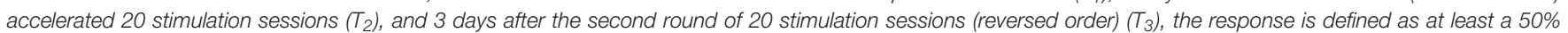

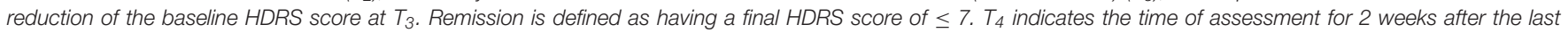
measurement, after $T_{3}$. These data were only collected for the aiTBS study. 
Although no significant differences between the two accelerated protocols were detected, $35 \%$ of the patients in the refractory depressed state responded, which was in line with the classical rTMS treatment response outcome for depression patients (Brunoni et al., 2017). For the remission rates, the $14 \%$ remission outcome seems rather low but is still in the range of results observed with the direct classical daily rTMS treatment, which is around 18.6\% (Berlim et al., 2014). Of note, although the remission rates remained unchanged for 2 weeks after the acute treatment phase for aHF-rTMS (personal communication), the remission rates doubled after 2 weeks for aiTBS stimulation (Duprat et al., 2016), suggesting that the response and remission rates may be delayed for aiTBS. This is not contradictive to the neurophysiological effects, which are assumed to be induced by iTBS (Chung et al., 2015). In the current stage III study, as a result of adding the two remitters 2 weeks after having ended the aiTBS stimulation protocol (see Table 1), the remission outcome for both accelerated protocols was $21 \%$.

It also seems that in the refractory depressed state, the response and remission rates are somewhat lower when including the less resistant depressed patients for the arTMS paradigms (Baeken et al., 2013: aHF-rTMS response: 35\%, remission: 35\%; Duprat et al., 2016: aiTBS response: 38\%, remission: 30\%), daily left DLPFC stimulation (Blumberger et al., 2018: HF-rTMS response: $47 \%$, remission: $27 \%$; iTBS response: $49 \%$, remission: 32\%), and daily dmPFC stimulation (Bakker et al., 2015: HFrTMS response: 50.6\% remission: $38.5 \%$; iTBS response: $48.5 \%$, remission: $27.9 \%$ ). However, given the limited number of large, placebo-controlled arTMS trials, this assumption is merely speculative at this point. Indeed, before taking firm conclusions for this retrospective stage III study, we clearly lacked sufficient power. To even find a small effect size of $f=0.10$ in an ANOVA, for a post hoc calculation of repeated measures of ANOVA with a total sample of 28 patients, the power to find a group difference is 0.19, which is rather low (calculated with G-Power 3.1.9.2.). To properly address the question of whether arTMS paradigms could be as successful as ECT in refractory depression, large, multicenter, randomized, non-inferiority, placebo-controlled clinical trials will be necessary to compare arTMS with ECT in such samples. The absence of clinical differential effects between the sham and the active accelerated treatment suggests some form of placebo effect. Placebo responses in depressed patients undergoing rTMS have been reported earlier (Berman et al., 2000), and hopes and beliefs for a new treatment approach, after numerous failed pharmacological and psychotherapeutic interventions (Mommaerts and Devroey, 2012), may add to a placebo effect. On the other hand, a recent meta-analysis showed that the placebo effect may be a component of rTMS therapeutic response (Razza et al., 2018). Nevertheless, the standard contrasting to the accelerated left DLPFC and HFrTMS treatment (Fitzgerald et al., 2018: standard response: $29.8 \%$, remission: $17.5 \%$; accelerated: $20.3 \%$, remission: $11.9 \%$ ) did not show any significant differences between the protocols, suggesting that standard daily and arTMS have similar clinical outcome rates in MDD.
Altogether, regardless of brain target, frequency, or coils, the response and remission rates do not seem to be that different between the rTMS paradigms. This also implies that within current safety parameters and stimulation guidelines, we may have reached a ceiling effect to treat depression, although with acceptable clinical results (compared to pharmacotherapy), but not with the outcomes of ECT. New types of coils, such as the $\mathrm{H}_{1}$ coil, as well as magnetic seizure therapy (MST) are used to improve the clinical outcome with some potential for higher remission rates (Harel et al., 2014; Cretaz et al., 2015), however, not with that magnitude to challenge the current ECT outcome rates. Individualization of the rTMS parameters has rightfully gained attention (Padberg et al., 2017) as Drysdale et al. (2017) were able to apply the machine learning algorithms (resting state $\mathrm{fMRI}$ ), identifying the four biotypes of depression and increasing the prediction of response after dmPFC stimulation to $93.6 \%$, when combining the connectivity and clinical features. Whether such scientific approaches will positively affect the future response and remission rates remains to be determined.

\section{CONCLUSION}

Applying the current rTMS parameters for depression patients seems to yield similar response and remission rates, regardless of the stimulation protocols used. So the major impact of the arTMS protocols is the important gain of time. Instead of daily application for 4-6 weeks, similar results are to be expected when concentrating all sessions over a couple of days. From a patient as well as from a medical view, the restriction on the duration of the depressive episode may be a major motivation to consider arTMS. However, any rTMS treatment protocol has to be considered as an acute treatment, and the improvement and maintenance of clinical effects should remain as a priority in the field of neurostimulation, further pursuing clinical outcomes challenging the successes of ECT in refractory depression, especially for remission.

\section{ETHICS STATEMENT}

The study were in accordance with the guidelines laid down in the Declaration of Helsinki (2004) and approved by the local ethics committee UZ Brussel and the local Ghent University Hospital. All subjects gave written informed consent.

\section{AUTHOR CONTRIBUTIONS}

$\mathrm{CB}$ collected and analyzed the data and wrote the manuscript.

\section{FUNDING}

This research was supported by a grant from the Scientific Fund W. Gepts and by the Ghent University Multidisciplinary Research Partnership "The integrative neuroscience of behavioral control." 


\section{REFERENCES}

Baeken, C., Marinazzo, D., Everaert, H., Wu, G. R., Van Hove, C., Audenaert, K., et al. (2015). The impact of accelerated HF-rTMS on the subgenual anterior cingulate cortex in refractory unipolar major depression: insights from 18FDG PET brain imaging. Brain Stimul. 8, 808-815. doi: 10.1016/j.brs.2015. 01.415

Baeken, C., Vanderhasselt, M. A., Remue, J., Herremans, S., Vanderbruggen, N., Zeeuws, D., et al. (2013). Intensive HF-rTMS treatment in refractory medication-resistant unipolar depressed patients. J. Affect. Disord. 151, 625631. doi: 10.1016/j.jad.2013.07.008

Bakker, N., Shahab, S., Giacobbe, P., Blumberger, D. M., Daskalakis, Z. J., Kennedy, S. H., et al. (2015). rTMS of the dorsomedial prefrontal cortex for major depression: safety, tolerability, effectiveness, and outcome predictors for 10 $\mathrm{Hz}$ versus intermittent theta-burst stimulation. Brain Stimul. 8, 208-215. doi: 10.1016/j.brs.2014.11.002

Berlim, M. T., van den Eynde, F., Tovar-Perdomo, S., and Daskalakis, Z. J. (2014). Response, remission and drop-out rates following high-frequency repetitive transcranial magnetic stimulation (rTMS) for treating major depression: a systematic review and meta-analysis of randomized, double-blind and shamcontrolled trials. Psychol. Med. 44, 225-239. doi: 10.1017/S003329171300 0512

Berman, R. M., Narasimhan, M., Sanacora, G., Miano, A. P., Hoffman, R. E., Hu, X. S., et al. (2000). A randomized clinical trial of repetitive transcranial magnetic stimulation in the treatment of major depression. Biol. Psychiatry 47, 332-337. doi: 10.1016/S0006-3223(99)00243-7

Blumberger, D. M., Vila-Rodriguez, F., Thorpe, K. E., Feffer, K., Noda, Y., Giacobbe, P., et al. (2018). Effectiveness of theta burst versus high-frequency repetitive transcranial magnetic stimulation in patients with depression (THREE-D): a randomised non-inferiority trial. Lancet 391, 1683-1692. doi: 10.1016/S0140-6736(18)30295-2

Brunoni, A. R., Chaimani, A., Moffa, A. H., Razza, L. B., Gattaz, W. F., Daskalakis, Z. J., et al. (2017). Repetitive transcranial magnetic stimulation for the acute treatment of major depressive episodes: a systematic review with network meta-analysis. JAMA Psychiatry 74, 143-152. doi: 10.1001/jamapsychiatry.2016. 3644

Chung, S. W., Hoy, K. E., and Fitzgerald, P. B. (2015). Theta-burst stimulation: a new form of TMS treatment for depression? Depress. Anxiety 32, 182-192. doi: 10.1002/da.22335

Cretaz, E., Brunoni, A. R., and Lafer, B. (2015). Magnetic seizure therapy for unipolar and bipolar depression: a systematic review. Neural. Plast. 2015:521398. doi: 10.1155/2015/521398

Drysdale, A. T., Grosenick, L., Downar, J., Dunlop, K., Mansouri, F., Meng, Y., et al. (2017). Resting-state connectivity biomarkers define neurophysiological subtypes of depression. Nat. Med. 23, 28-38. doi: 10.1038/nm.4246

Duprat, R., Desmyter, S., Raedt de, R., van Heeringen, K., Van den Abbeele, D., Tandt, H., et al. (2016). Accelerated intermittent theta burst stimulation treatment in medication-resistant major depression: a fast road to remission? J. Affect. Disord. 200, 6-14. doi: 10.1016/j.jad.2016.04.015

Fitzgerald, P. B., Hoy, K., McQueen, S., Maller, J. J., Herring, S., Segrave, R., et al. (2009). A randomized trial of rTMS targeted with MRI based neuro-navigation in treatment-resistant depression. Neuropsychopharmacology 34, 1255-1262. doi: $10.1038 /$ npp.2008.233

Fitzgerald, P. B., Hoy, K. E., Elliot, D., Susan McQueen, R. N., Wambeek, L. E., and Daskalakis, Z. J. (2018). Accelerated repetitive transcranial magnetic stimulation in the treatment of depression. Neuropsychopharmacology 43, 1565-1572. doi: 10.1038/s41386-018-0009-9
Fox, M. D., Halko, M. A., Eldaief, M. C., and Pascual-Leone, A. (2012). Measuring and manipulating brain connectivity with resting state functional connectivity magnetic resonance imaging (fcMRI) and transcranial magnetic stimulation (TMS). Neuroimage 62, 2232-2243. doi: 10.1016/j.neuroimage.2012.03.035

George, M. S., and Post, R. M. (2011). Daily left prefrontal repetitive transcranial magnetic stimulation for acute treatment of medication-resistant depression. Am. J. Psychiatry 168, 356-364. doi: 10.1176/appi.ajp.2010.10060864

Hamilton, M. (1967). Development of a rating scale for primary depressive illness. Br. J. Soc. Clin. Psychology. 6, 278-296. doi: 10.1111/j.2044-8260.1967.tb00530.x

Harel, E. V., Rabany, L., Deutsch, L., Bloch, Y., Zangen, A., and Levkovitz, Y. (2014). H-coil repetitive transcranial magnetic stimulation for treatment resistant major depressive disorder: an 18-week continuation safety and feasibility study. World J. Biol. Psychiatry 15, 298-306. doi: 10.3109/15622975. 2011.639802

Holtzheimer, P. E. III, McDonald, W. M., Mufti, M., Kelley, M. E., Quinn, S., Corso, G., et al. (2010). Accelerated repetitive transcranial magnetic stimulation for treatment-resistant depression. Depress. Anxiety 27, 960-963. doi: 10.1002/ da. 20731

Kennedy, S. H., and Giacobbe, P. (2007). Treatment resistant depressionadvances in somatic therapies. Ann. Clin. Psychiatry 19, 279-287. doi: 10.1080/ 10401230701675222

Lefaucheur, J. P., André-Obadia, N., Antal, A., Ayache, S. S., Baeken, C., Benninger, D. H., et al. (2014). Evidence-based guidelines on the therapeutic use of repetitive transcranial magnetic stimulation (rTMS). Clin. Neurophysiol. 125, 2150-2206. doi: 10.1016/j.clinph.2014.05.021

Mommaerts, J. L., and Devroey, D. (2012). The placebo effect: how the subconscious fits in. Perspect. Biol. Med. 55, 43-58. doi: 10.1353/pbm.2012.0005

Padberg, F., Brem, A. K., Palm, U., Pogarell, O., Hasan, A., Brunelin, J., et al. (2017). Discovering the individual brain: brain stimulation in psychiatry: editorial I to the supplement from the 2nd European conference on brain stimulation in psychiatry. Eur. Arch. Psychiatry Clin. Neurosci. 267, 109-112. doi: 10.1007/ s00406-017-0853-3

Razza, L. B., Moffa, A. H., Moreno, M. L., Carvalho, A. F., Padberg, F., Fregni, F., et al. (2018). A systematic review and meta-analysis on placebo response to repetitive transcranial magnetic stimulation for depression trials. Prog. Neuropsychopharmacol. Biol. Psychiatry 81, 105-113. doi: 10.1016/j.pnpbp. 2017.10.016

Rush, A. J., and Siefert, S. E. (2009). Clinical issues in considering vagus nerve stimulation for treatment-resistant depression. Exp. Neurol. 219, 36-43. doi: 10.1016/j.expneurol.2009.04.015

Rush, A. J., Thase, M. E., and Dubé, S. (2003). Research issues in the study of difficult-to-treat depression. Biol. Psychiatry 53, 743-753. doi: 10.1016/S00063223(03)00088-X

Ward, M. P., and Irazoqui, P. P. (2010). Evolving refractory major depressive disorder diagnostic and treatment paradigms: toward closed-loop therapeutics. Front. Neuroeng. 3:7. doi: 10.3389/fneng.2010.00007

Conflict of Interest Statement: The author declares that the research was conducted in the absence of any commercial or financial relationships that could be construed as a potential conflict of interest.

Copyright (c) 2018 Baeken. This is an open-access article distributed under the terms of the Creative Commons Attribution License (CC BY). The use, distribution or reproduction in other forums is permitted, provided the original author(s) and the copyright owner(s) are credited and that the original publication in this journal is cited, in accordance with accepted academic practice. No use, distribution or reproduction is permitted which does not comply with these terms. 